\title{
Experiment Proposal on How Different Types of Mood Affect ISE
}

\author{
Qi Jun Zhao \\ Shanghai Starriver Bilingual Highschool, Shanghai, 200000 Shanghai, China. \\ Email:1515290223@qq.com
}

\begin{abstract}
This experiment aims to find out the impact mood has on short term ISE and how ESE mediates this impact. Three types of mood would be included: Ability related mood are primarily caused by oneself; luck related by the environment; and empathetic by observing others. Each of the three mood types will also have positive and negative versions. A pretest would adjust each mood manipulation, so they induce the same mood strength. In the main experiment, participants would first take an ISE test, then they will be randomly assigned to one of six mood manipulations, then take another ISE test, then an ESE test. This experiment would use the name letter test to measure ISE. The data of the relationship between mood manipulation type, ISE change, and ESE would be analyzed. It is predicted that situations that relates closely to oneself will have more impact on ISE.
\end{abstract}

Keywords: Implicit self-esteem, Explicit self-esteem, Mood types

\section{INTRODUCTION}

Self-esteem is defined as the degree to which the qualities and characteristics contained in one's selfconcept are perceived to be positive in APA dictionary [1]. Implicit self-esteem is defined in this paper as the part of self-esteem that is unconscious. Trait ISE is the baseline around which state ISE fluctuates. This paper examines how different types of situations affect state ISE, while controlling for the strength of mood different situations cause. There had been past research studying how explicit self-esteem mediates the relationship between events and ISE or ISE related constructs. The following the summaries of two related experiments. The first is titled "Fluctuations in state implicit self-esteem in response to daily negative events" by Tracy DeHart and Brett W. Pelham. (DeHart, Pelham, 2007) [2] The researchers first gave participants a state ESE and selfconcept clarity questionnaire. Then, each day for the next three weeks, participants filled out four questionaries: A state implicit self-esteem questionnaire using the name letter test; a questionnaire checking for positive and negative life events (i.e., Did something embarrassing happen; Did you work towards completing an assignment); a mood questionnaire; and a state explicit self-esteem questionnaire. The results include that ESE predicts a level of ISE which fluctuates based on daily negative events. High ESE means a high level of base ISE and that higher daily negative events increase ISE. Low ESE means a low level of base ISE and that higher daily negative events decrease ISE. Fluctuation of ISE score from negative to positive events is about $10 \%$ for low ESE individuals and $-3.5 \%$ for high ESE individuals. Another study by Jonathon D. Brown and Tracie A. Mankowski titled Self-Esteem, Mood, and SelfEvaluation: Changes in Mood and the Way You See You (Brown, Mankowski, 1991) [3] focused on selfevaluations and mood. The researchers manipulated mood in two experiments by letting participants read affect charged sentences and listen to affect charged music, and the third part of the experiment uses questionnaire to access mood in daily life. The researchers found a similar relationship between explicit self-esteem, mood manipulation, and self-evaluations as the previous experiment. Explicit self-esteem determines a baseline on which self-evaluation fluctuates due to mood manipulations, high ESE correlates with a high baseline self-evaluation. When people experience negative mood, people with high ESE experience increase in self-evaluations while people with low ESE experience decreased self-evaluations.

This paper proposes an experiment that provides experimental evidence on how different types of mood manipulations affect state ISE, and how ESE affects this relationship. This experiment differs from the first summarized study because this experiment provides 
experimental evidence while the first study provides correlational evidence. This experiment differs from the second summarized experiment because this experiment divides mood into three mood types.

\section{SIX MOOD MANIPULATIONS}

The situations we encounter in life are very complex and can affect ISE in a multitude of ways. In this experiment the focus is on situations that induce mood, so different types of situations would be called mood types. In this experiment mood is split into three types: Abilities related, luck related, and empathetic related. We can feel mood either for ourselves or for empathetic feelings towards others. The situation that caused the mood we feel for ourselves can either happen primarily because of something we did or primarily because of external factors. The mood we feel for ourselves caused by something we did is called ability related; if the mood is caused by external forces it is called luck related. If the mood is felt for others, it is called empathetic related. There would be three kinds of mood manipulations in the study, each corresponding with a mood type. Each mood manipulation would be further split into positive and negative mood manipulations. The specific mood manipulations are the following: In the ability related mood manipulation, participants would be given some information about a coffee machine, which they would then use to make a presentation to sell that machine. They would present to some judges and receive a score and some feedback. The judges would score them about 90 out of 100 and give comments of praise for the positive manipulation. The judges would score them about 50 out of 100 and give them harsh criticisms for the negative manipulation. In the luck related manipulation, participants would either be told that they won a 100dollar gift card or the lie that the final test involves them doing a lot of exhausting exercise. For the empathetic related manipulation, participants would either watch a 10-minute film of others experiencing a happy event or a sad event, they would be asked to rate the films aesthetically though the scores they give wouldn't matter for the experiment. The purpose of filling out the aesthetics questionnaire is to conceal the reason that they watched the film to induce mood.

\section{PRE-TEST}

Different mood manipulations would induce mood of different strength. This experiment aims to find out how different mood types affect ISE, so mood strength is a confounding variable, and it needs to be controlled. In the pre-test, a separate group of participants would be recruited and given a random mood manipulation. After that, they would take a questionnaire asking about their mood (Positive and Negative Affectivity Schedule (PANAS)). Parameters in the mood manipulation test would be adjusted to try to keep the strength of the mood induced the same. For example, if in the positive ability manipulation participants reported significantly higher mood on average than participants in the other two positive manipulations, then instead of being told that they scored a 95 they would be told that they scored a 90 . A total of three pre-tests would be run, with one adjustment following each of the three pre-tests. After three pretests and adjustments the mood strength each mood manipulation induce should be about the same under ideal circumstances. The three positive manipulations should induce the same mood strength, and the three negative manipulations should induce the same mood strength, and the absolute value of all six manipulations' induced mood strength should be about the same.

\section{MAIN TEST}

Participants would be invited into the lab. They would take a name letter test [4]. They would be told to trust their intuition and make decisions quickly. They would be told that the test is used for linguistic studies. This procedure is alike to the procedure used in (Tracy DeHart, Brett W. Pelham,2006) [2]. After the NLT, participants would be randomly assigned to one of six mood manipulation tasks. Participants should not be aware that the mood manipulation tasks have the purpose of inducing mood. After the mood manipulation task, participants would take another NLT. They should take the second NLT 5 minutes after the mood manipulation had occurred. This should eliminate time as a confounding variable. After taking the second ISE test, the participants would take an explicit self-esteem measure using the Rosenberg's self-esteem scale. The participants would be debriefed about the lies that were told and the true purpose of the experiment, and the experiment is finished.

\section{PRETEST DATA ANALYSIS}

In the pretest, after each test, the average of the mood strength score in each manipulation would be taken. An average of all mood strength score across all manipulations would be taken. Parameters in different mood manipulations would be adjusted to try to get the mood strength induced closer to the average of all mood strength scores. A one-way ANOVA would be used after each test. In this case, a null hypothesis validation would be ideal because keeping the mood strength across different manipulation the same means that the confounding variable of mood strength is eliminated.

\subsection{Main Test Data analysis}

In the main test, the NLT data would be converted into an ISE score. The scores the participants gave to their first letter in their first and last names would be averaged. This average would be standardized using an outside data pool 
of rating of letters to form an $\mathrm{z}$ score. This should reflect how much standard deviations the participants liked their name letters more or less than the average liking of the letters. This would be their ISE score.

The first ISE score would be correlated with ESE to see if there's a strong correlation using the Pearson's $r$ correlation test. In the main test, a one-way repeated ANOVA would be run, since it is a three by two design. The first ISE score, the mood manipulation type, and the second ISE score would be used in the one-way repeated ANOVA test. After that, low ESE and high ESE data would be separated, and a one-way ANOVA would be performed on each group.

\subsection{Predicted Results}

Note these are predicted, not real results. No real experiments have been run.

Based on past research it is predicted that there is a strong correlation between ESE and ISE. For the main test, the prediction is that ability related mood causes a stronger impact on ISE than luck related mood and luck related mood cause a stronger impact on ISE than empathy related mood. This is the prediction for both positive and negative mood. In conclusion, the more a situation relates to oneself, the more impact on ISE it has. Relation to oneself and mood are two variables that affect ISE, and keeping mood the same, relation to oneself could predict ISE. When the results are analyzed separately in groups of high ESE and low ESE participants, it is predicted that the baseline ISE would be positively correlated with ESE. For both groups, the positive mood would increase in ISE. When negative mood is induced, low ESE participants would experience a decrease in ISE, but high ESE participants would experience an increase in ISE. It is predicted that overall, the effect on short term ISE would be high.

Negative manipulation predicted results graph. Note these are predicted, not real results. No real experiments have been run.

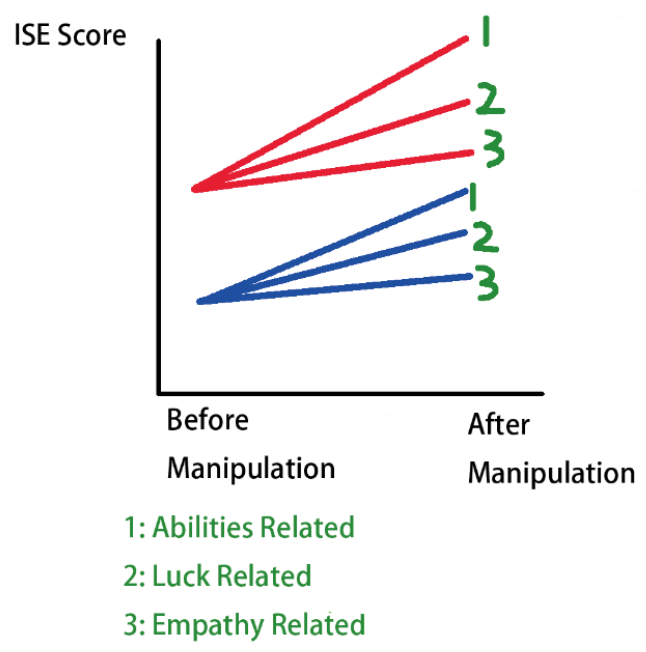

Figure 1. Positive manipulation predicted Results.

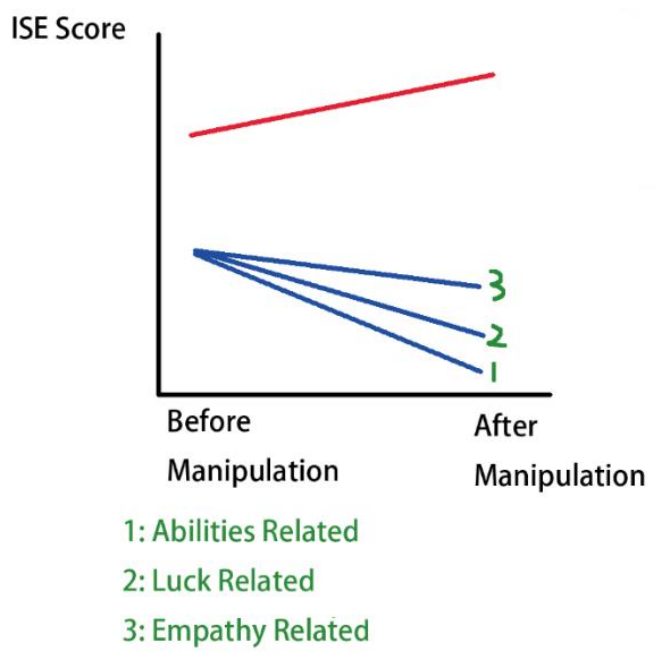

Figure 2. Negative manipulation predicted Results. 
Whether a stronger type of mood manipulation would cause a stronger increase in ISE for the ESE group cannot be predicted so it is not specified in the graph.

\section{CONCLUSION}

The following is a theory for understanding. These claims are not proved by experiments. ISE is how positively we perceive ourselves unconsciously. State ISE is ISE at every moment. State ISE may fluctuate based on different events. Events can be described in two dimensions: How much mood they cause; How closely it is related to one's competence. The more mood an event cause, or the more closely related an event is to oneself, the more it will change state ISE. For people with high ESE, however, a negative mood would increase state ISE. Some previous experiments did not use the second dimension proposed here to classify events.

This experiment proposal uses six mood manipulations to find out whether the connection between the event and one's competence influences ISE. There are several limitations to this study. First, the same mood manipulation could result in different strength of mood induced for different individuals. Second, the name letter test is proved to have validity issues according to a metanalysis (Buhrmester, Hart Blanton, Swann 2011) [5]. Third, the mood types that is defined in this study is too clear cut. In a natural setting there could be multiple components of a mood inducing situation. Fourth, there isn't a scale to measure how much an event relates to one's competence. This makes it hard to calculate the correlation between how much an event relates to one's competence to change in state ISE. Different ISE measures like the IAT or the endowment effect could be used to measure ISE after mood manipulation. More specific constructs could be identified to determine what affects state ISE in daily life. Future research could use mood strength as the independent variable while controlling for mood type to find out how mood strength affects ISE. Future research could try to plot ISE against time, for example, what happens to ISE each minute after the mood induction for twenty minutes.

\section{REFERENCES}

[1] APA Dictionary of Psychology, Self Esteem.https://dictionary.apa.org/self-esteem

[2] T. DeHart, B.W. Pelham, Journal of Experimental Social Psychology 43 (2007) 157-165

[3] Jonathon D. Brown \& Tracie A. Mankowski, personality processes and individual differences, (1991)

[4] M. Nuttin Narcissism beyond Gestalt and awareness: The name letter effect (1985)

[5] Michael D. Buhrmester \& Hart Blanton \& William B.
Swann, Jr, Journal of Personality and Social Psychology (2011) 